\title{
Nutritional Analysis of Biovalorisaton of Homogeneous Food Processing By-products
}

\author{
Winnie Faustinelie ${ }^{1}$, Ken Chi-Lik Lee ${ }^{1}$ \\ ${ }^{1}$ Nanyang Technological University, 21 Nanyang Link, School of Physical and Mathematical \\ Sciences, Singapore 637371, Singapore
}




\begin{abstract}
Okara and Brewer's Spent Grain (BSG), the food by-products generated during soybean and beer production, respectively, are two of the many food processing side streams that are suitable for human consumption but often discarded. Okara and BSG offer good amounts of both macro and micronutrients. More importantly, they are an exceptional source of proteins. Solid-state fermentation was used to enhance the bioavailability of their intrinsic nutrients. In this study, eight unique strains of Aspergillus Oryzae were used to ferment both Okara and BSG. Subsequently, various quantitative and qualitative analyses were conducted to determine the Total Polyphenolic Content (TPC) and Total Flavonoids Content (TFC) of the fermented biomasses. TPC and TFC were determined via spectroscopic analysis. The microbial fermentation of Okara by Black Koji resulted in the highest increase of TPC and TFC (5- and 13-fold respectively), compared to the control Okara. Meanwhile for BSG, the microbial fermentation by M-1 resulted in the highest increase of TPC in BSG (7-fold).
\end{abstract}




\section{ACKNOWLEDGEMENT}

I would like to express my gratitude to people who had supported me throughout my journey in doing Final Year Project. First and foremost, I would like to thank Dr. Ken Lee, my supervisor, who has patiently guided me and given me the opportunity to work on this project. I have learned a lot through this opportunity, be it research or presentation skills, knowing the purpose of doing all these things. This challenging project might not be successfully done without all the continuous support and encouragement by my supervisor and his group, KRG members. Thank you for all the advice and help given to me whenever I faced any doubts or difficulties. Last but not least, I would like to thank Sing Ghee Beancurd Manufacturer and Brewerkz for providing material support to this project. 


\section{TABLE OF CONTENT}

1. Introduction......................................................................5

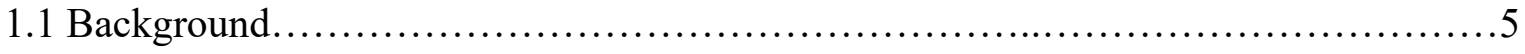

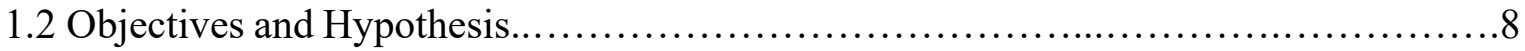

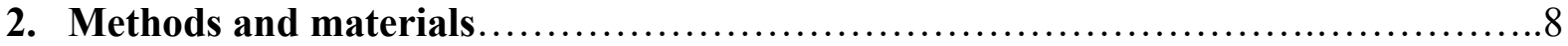

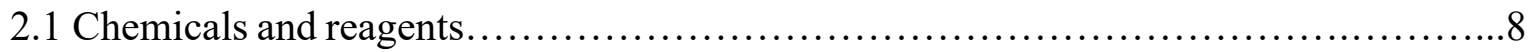

2.2 Fungi, Okara and Brewer's Spent Grain (BSG) ...............................

2.3 Solid State Fermentation of Okara and BSG...................................9

2.4 Total Polyphenolic Content (TPC) and Total Flavonoids Content (TFC)............. 10

2.5 Statistical Analysis.......................................................... 11

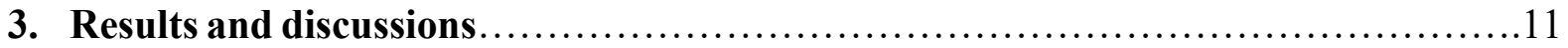

3.1 Calibration Curve and Linearity for Quantification.............................. 11

3.2 Total Polyphenolic Compound (TPC) Content................................ 12

3.3 Total Flavonoids Compound (TFC) Content ................................ 14

4. Conclusion and future work ................................................. 16

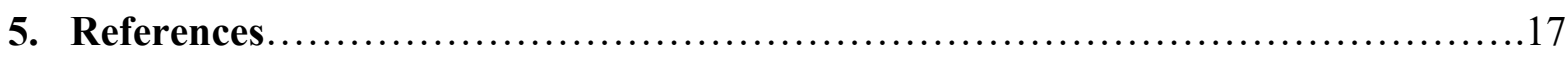




\section{INTRODUCTION}

\subsection{Background}

In recent years, there have been growing concerns over food waste. Food waste has farreaching impacts on the economy, environment, and society. ${ }^{1}$ Food processing and manufacturing companies are recognized to contribute to a significant fraction of the total food waste generated. ${ }^{2}$ In Singapore, the amount of food waste generated has increased by approximately 20 percent over the past 10 years, alongside the increasing population and economic activity. ${ }^{2}$ This has a significant impact on Singapore's ability to ensure food security as $90 \%$ of the total food supply is imported. ${ }^{3}$ Food waste can be categorized into two types: avoidable and unavoidable. The former can be prevented through intentional consumption, whereas the latter consists of food unfit for consumption. ${ }^{4}$ Therefore, this project aims to reduce unavoidable food waste by upcycling them into food that is fit for human consumption.

Homogeneous food wastes, such as Okara and Brewer's Spent Grain (BSG), by-products of soy milk or bean curd, and beer, respectively, are generated in large amounts due to their high consumption worldwide. Approximately $1.1 \mathrm{~kg}$ of raw Okara is produced for every 1 $\mathrm{kg}$ of soybean processed into soy milk or bean curd. As a result, about 10000 tons of Okara is amassed in Singapore alone annually. ${ }^{5,6}$ As for BSG, around $20 \mathrm{~kg}$ of BSG is produced for every $100 \mathrm{~L}$ of beer manufactured. ${ }^{7}$ Oftentimes, these food wastes serve as animal feed, while the remainder is incinerated due to their high perishability. The disposal of such "high nutrient" wastes might cause unwanted environmental problems; the production of odours and leachates in particular. 8,9

In recent years, generating high value-added bio-products (e.g., amino acids, polyphenols, flavonoids, etc.) using microorganisms has been the research focus of many biotechnological industries. ${ }^{10}$ The study involving "nutrient-rich" biomass by Solid-State Fermentation (SSF) is of particular interest. In this study, eight different strains of Aspergillus Oryzae will be used as the starter microbes to initiate the SSF. Each of the different strains has a different purpose in food preparation; SP-01 and SP-05 fungi for soy sauce production, M-1 and EM-2 fungi for miso production, NJK110 and Fuji fungi for sake production, and Black Koji for shochu production in Japan. Solid-State Fermentation is a simple and efficient approach to enhance the functional properties of many biomass's nutraceutical values. ${ }^{11}$ Okara and $\mathrm{BSG}$ are recognized to possess predominantly 
inaccessible bioactive components and are highly perishable, which prevent them from being a viable food source. ${ }^{5,9}$ The application of SSF can induce many changes to the physicochemical and bioactivity of the substrates, which results in enhanced nutritional quality. ${ }^{11}$ Consumption of fermented products are believed to impart numerous health benefits (e.g., hypertension, high cholesterol, diabetes, etc). ${ }^{5}$

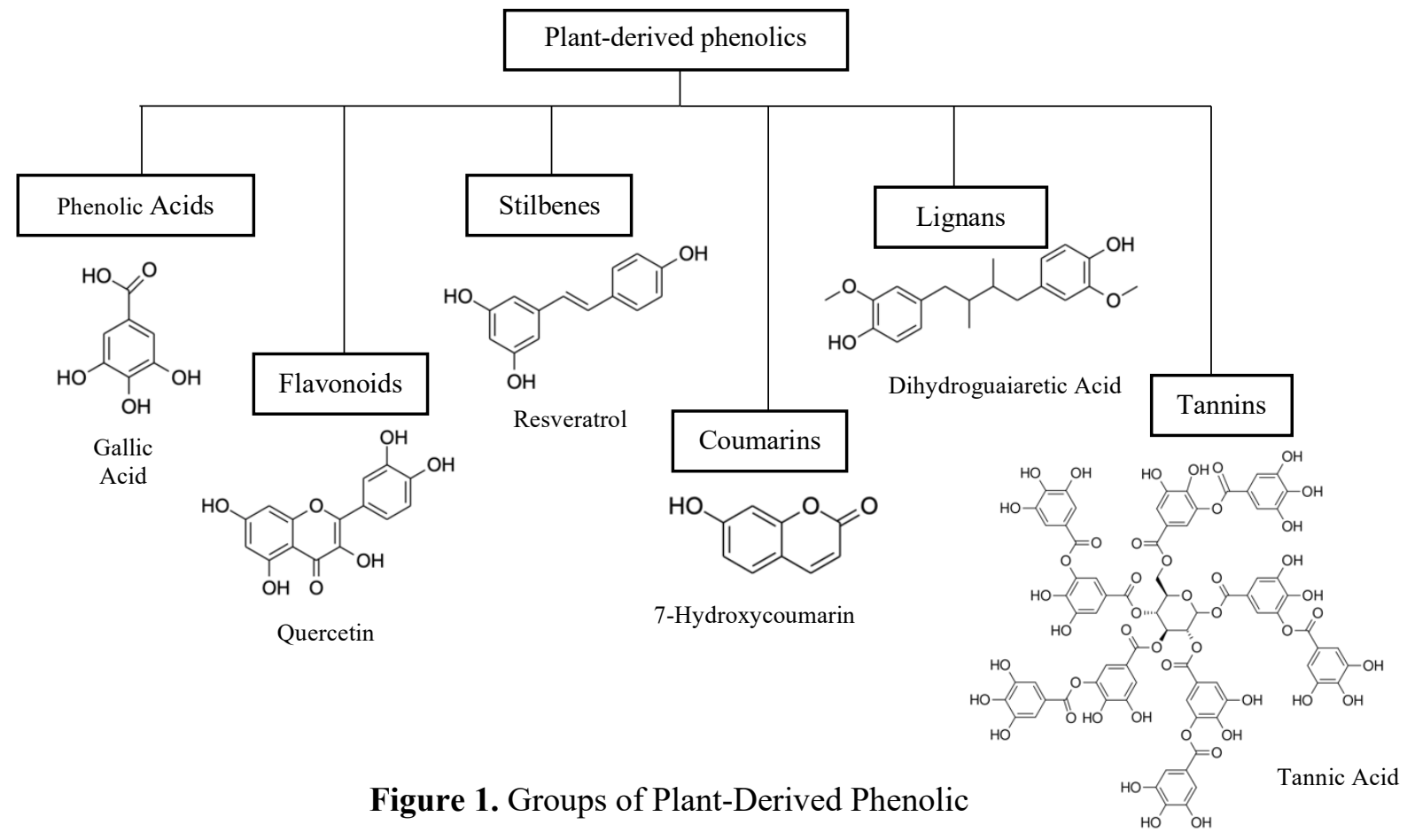

In this study, we will be focusing on antioxidant compounds, such as polyphenols and flavonoids. As shown in Figure 1, phenolic compounds consist of an extensive range of substances that possess aromatic ring bearing with one or more hydroxyl substituents. In natural form, polyphenols vary from simple molecules (e.g., phenolic acids) to highly polymerized molecules (e.g., tannins).

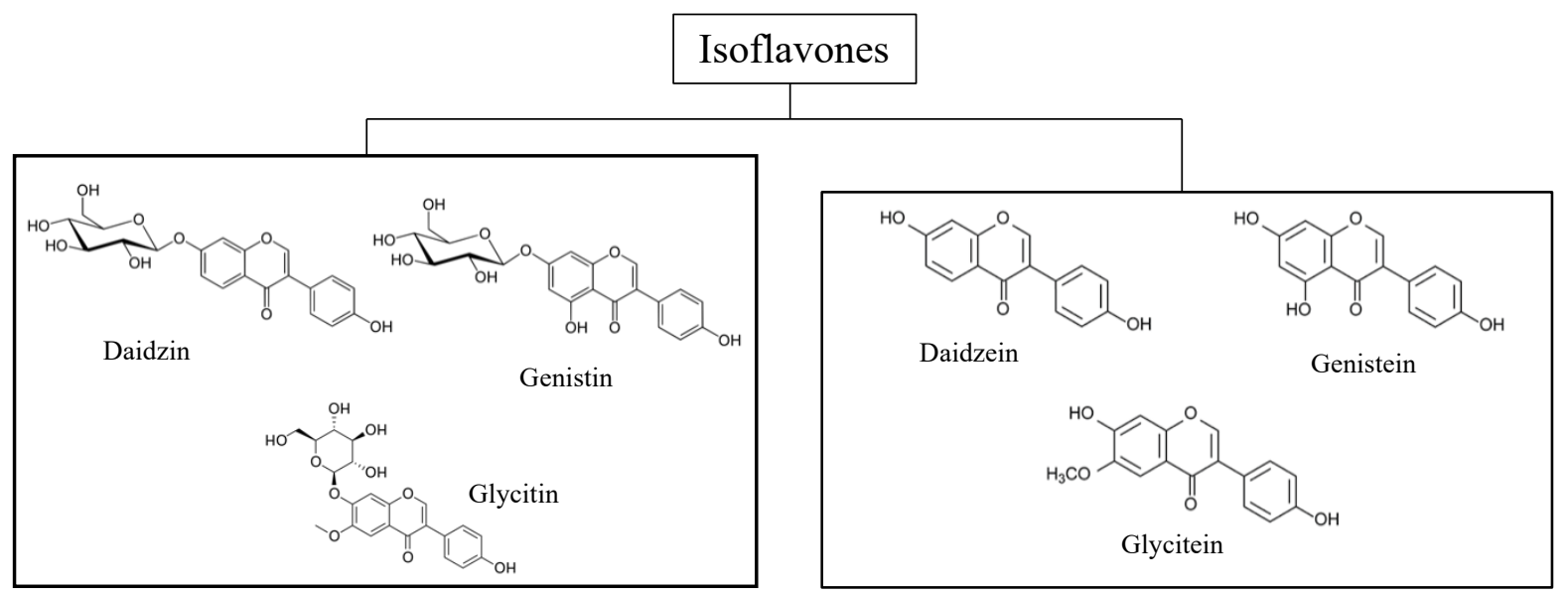

Isoflavones $\beta$-Glucoside

Isoflavones Aglycones

Figure 2. Groups of Isoflavones 
Isoflavones, a flavonoid, were highlighted to possess various biological functions (e.g., antioxidant, anti-carcinogenic, reduction of cholesterol levels, estrogenic activity, etc). ${ }^{12}$ Based on the World Health Organization statistics, age-related diseases, especially cardiovascular disease, accounted for $31 \%$ of all death worldwide. ${ }^{13}$ It is estimated that 17.9 million people suffered from cardiovascular diseases every year. Recent epidemiologic evidence has suggested that higher flavonoid intake would help in reducing coronary heart disease mortality. ${ }^{14}$<smiles>Cc1ccc(O)c(O)c1</smiles><smiles>[R]C(C)C</smiles><smiles>Cc1ccc(O)c([O-])c1</smiles><smiles>[R]</smiles>

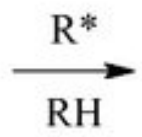<smiles>CC1=CC(=O)C(=O)C=C1</smiles>

Scheme 1. Flavonoids free-radical scavenging mechanism

As seen in Scheme 1, these antioxidant compounds scavenge free radicals, such as superoxide, peroxyl, and hydroperoxide, and therefore inhibit the oxidative mechanisms that lead to degenerative diseases. ${ }^{15}$ Studies have shown that reactive oxygen species reacted with free radicals generated during cellular metabolism could play a significant role in chronic disease pathogenesis, including cancer and coronary heart disease. ${ }^{16}$ Hence, dietary antioxidants can act as an inhibitor in combating the free radicals and reactive oxygen species, thereby reducing the risk of chronic diseases. Therefore, in this study, we are interested in evaluating the effectiveness of fermentation using various food-grade microorganisms in increasing TPC and TFC.

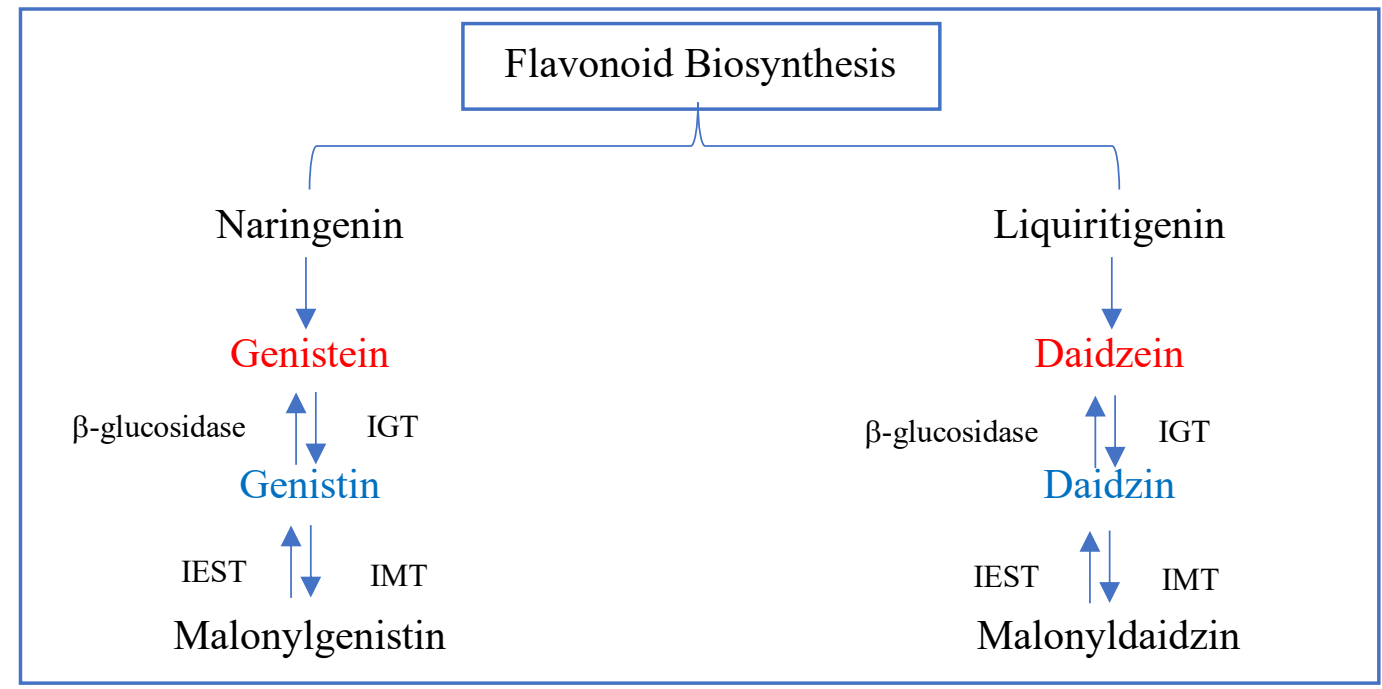

Scheme 2. The metabolic pathway for the biosynthesis of flavonoids. Key:

IGT, isoflavone 7-O-glycosyltransferases; IMT, isoflavone glucoside malonyltransferase; IEST, isoflavone malonylglucoside malonylesterase 
By analyzing the flavonoid content of pre-fermentation and post-fermentation with Aspergillus Oryzae, we can further expand our understanding of the metabolic transformation during the fermentation process. Scheme 2 displays the biochemical pathway taken by the subject of interest (i.e., flavonoids biosynthesis) during the microbial fermentation process. Isoflavones have been the topic of interest of many, which are known to be present in non-fermented foods in their glucoside forms (i.e., Genistin and Daidzin). ${ }^{5}$ It is believed that the aglycones form of isoflavones (i.e., Genistein and Daidzein) possess higher biological activities than their glucoside forms. They were believed to be more beneficial as natural isoflavonoid supplements for antidiabetic therapeutic use. The aglycone and glycosidic forms of isoflavones are interchangeable, and $\beta$-glucosidases catalyze the conversion of isoflavone glucosides to their biologically active aglycone form by hydrolyzing $\beta$-glucoside bond between the attached sugar moiety and the aglycone isoflavone. An illustration of the reaction mentioned above can be seen in Scheme 3. The fungal microbial activity activated the production of $\beta$-glucosidases, leading to biotransformation of phenolic compounds to their desired forms.

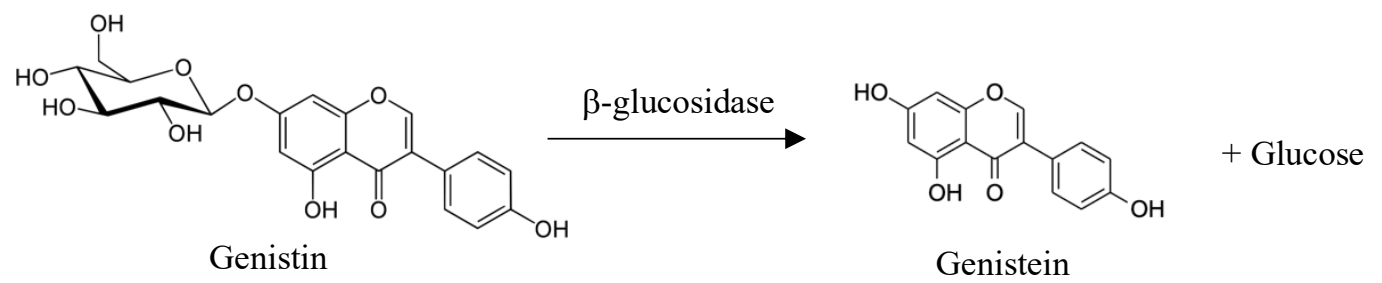

Scheme 3. Sample Illustration of Biotransformation of $\beta$-glucoside form (Genistin) to Aglycone form (Genistein)

\subsection{Objectives and Hypothesis}

This study aims to evaluate the effectiveness of the different strains of microbe in enhancing the nutritional value of BSG and Okara through the analysis of Total Polyphenolic Content (TPC) and Total Flavonoids Content (TFC). SSF is hypothesized to play a significant role in improving the TPC and TFC of Okara and BSG.

\section{MATERIALS AND METHODS}

\subsection{Chemicals and Reagents}

Gallic acid and Quercetin as standard were purchased from Sigma Aldrich. All other chemicals and reagents used, including Aluminum Chloride $\left(\mathrm{AlCl}_{3}\right)$, Potassium Acetate $\left(\mathrm{CH}_{3} \mathrm{COOK}\right)$, Sodium Carbonate $\left(\mathrm{Na}_{2} \mathrm{CO}_{3}\right)$, and Folin\&Ciocalteu's Phenol Reagent (FC), were obtained from commercial sources. 


\subsection{Fungi, Okara and Brewer's Spent Grain (BSG)}

Various strains of Aspergillus Oryzae, including SP-01, NJK110, AOH, Fuji, SP-05, EM2, M-1 and Black Koji were purchased from Nihon Jyozo Kogyo Co. and Akita Konno Shoten (Japan). Fresh Okara was obtained from Sing Ghee Beancurd Manufacturer (Singapore), while BSG was provided by Brewerkz (Singapore). Both fresh Okara and BSG were packed and stored in the freezer at $-20^{\circ} \mathrm{C}$ before use.

\subsection{By-product Solid-State Fermentation (SSF)}
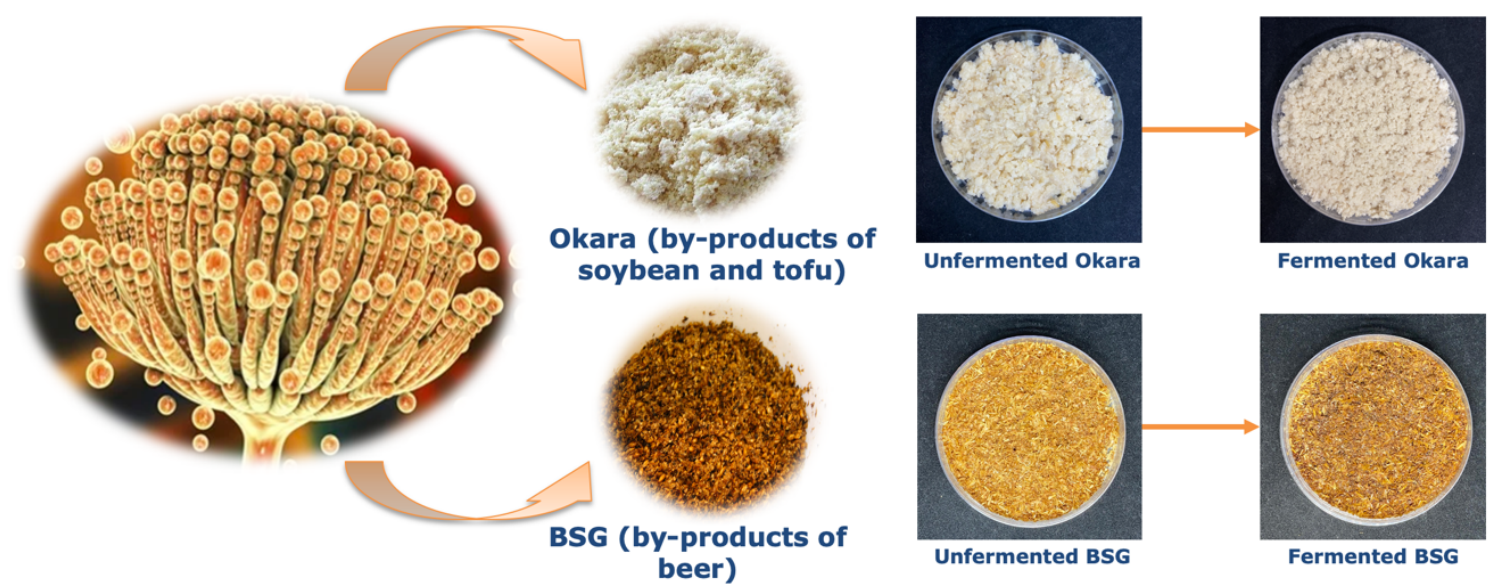

Figure 3. Solid-state fermentation on Okara and BSG using Natto

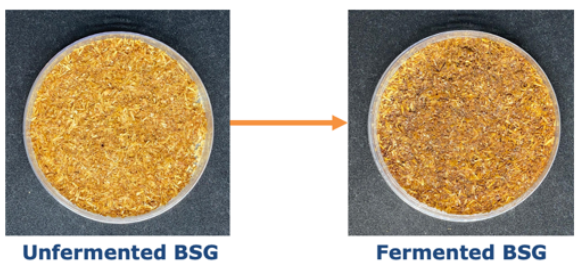

Figure 3. Solid-state fermentation on Okara and BSG using Natto

Defrosted Okara and BSG were autoclaved at $121^{\circ} \mathrm{C}$. BSG was subsequently dried and ground. $20 \mathrm{~g}$ of Okara were added into a petri dish, while $8 \mathrm{~g}$ of BSG were added into a petri dish and topped up with $12 \mathrm{~mL}$ of water to ensure sufficient moisture content (80\%). The biomasses were inoculated with a microbial loading $10^{6} \mathrm{spores} / \mathrm{g}$ for all types of fungus. The fermentation for all the samples was carried out at room temperature for four days, except for samples inoculated with Natto, which were incubated at $38^{\circ} \mathrm{C}$ for four days. Illustration of fermentation result can be seen in Figure 3, where fermented samples are generally darker in colour. Unfermented Okara and BSG were also prepared as control samples to ensure the absence of contamination. Afterwards, fermented samples were dried, ground, and stored in $4^{\circ} \mathrm{C}$ fridge before analysis. 


\subsection{Total Polyphenolic Content (TPC) and Total Flavonoids Content (TFC)}

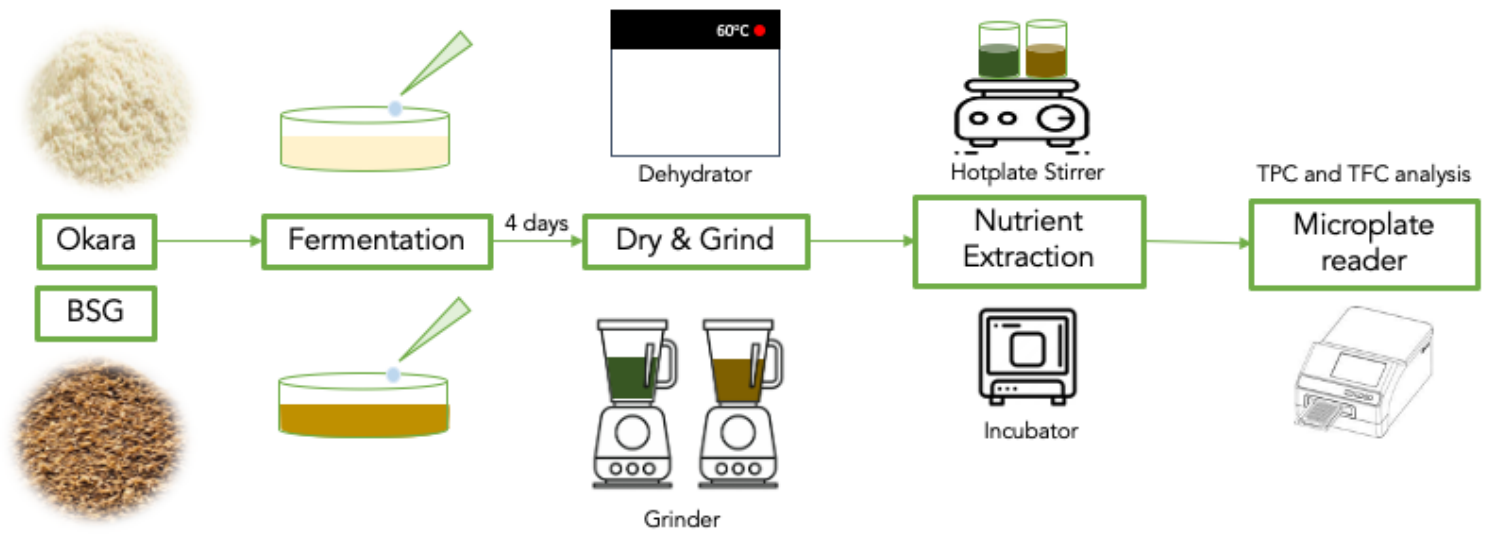

Figure 4. Illustration of the experimental process for TPC and TFC analysis using microplate reader

The overall experimental process is shown in Figure 4. TPC and TFC were extracted with green solvents ${ }^{17}$ (i.e., Methanol and Ethanol), and their absorbance at a specific wavelength were measured using a Monochromator Microplate Reader (Tecan Infinite M2000).

\subsubsection{TPC Analysis}

TPC was extracted by adding $100 \mathrm{mg}$ of the sample into $1 \mathrm{~mL}$ of $50 \%$ methanol $(\mathrm{v} / \mathrm{v})$. The mixture was mixed well before undergoing serial dilution (x10) if the sample is too concentrated. $100 \mu \mathrm{L}$ of the sample after serial dilution was mixed with $1 \mathrm{~mL}$ of FC solution and $800 \mu \mathrm{L}$ of $1 \mathrm{M} \mathrm{Na}_{2} \mathrm{CO}_{3}$. Eventually, it was left to heat at $45^{\circ} \mathrm{C}$ for 15 minutes, and the absorbance was measured using a microplate reader at wavelength $760 \mathrm{~nm}$ with Gallic Acid as the standard.

The gallic acid stock solution was prepared by dissolving $0.5 \mathrm{~g}$ of Gallic Acid in 10 $\mathrm{mL}$ of methanol and topped up to $100 \mathrm{~mL}$ using distilled water. Different concentrations ( 0 to $100 \mathrm{ppm}$ ) of gallic acid were prepared via serial dilution with distilled water to obtain the desired concentration. Thereafter, the absorbance of different Gallic acid solution concentrations was measured at $760 \mathrm{~nm}$ to obtain the calibration curve for TPC analysis.

\subsubsection{TFC Analysis}

$0.5 \mathrm{~g}$ of each sample was added to $3.5 \mathrm{~mL}$ of $80 \%$ ethanol $(\mathrm{v} / \mathrm{v})$ and left to soak at room temperature for 1 hour with frequent agitation. The mixture was then incubated 
at $40^{\circ} \mathrm{C}$ for 4 hours with shaking. Afterwards, the suspension was centrifuged for 20 minutes at $4000 \mathrm{rpm}$, and the supernatant was collected and filtered through $0.2 \mu \mathrm{m}$ syringe filters. $0.1 \mathrm{~mL}$ of aluminium chloride $\left(\mathrm{AlCl}_{3}, 10 \%\right), 0.1 \mathrm{~mL}$ of potassium acetate $\left(\mathrm{CH}_{3} \mathrm{COOK}, 1 \mathrm{M}\right)$ and $4.3 \mathrm{~mL}$ of $80 \%$ Ethanol were subsequently added to $0.5 \mathrm{~mL}$ of extract. The mixture was then incubated at room temperature for 40 minutes, and the absorbance was measured at $415 \mathrm{~nm}$ with Quercetin as the standard.

Quercetin stock solution was prepared by dissolving $5 \mathrm{mg}$ of dry Quercetin in $1 \mathrm{~mL}$ of Ethanol. Different concentrations were prepared by serial dilution using Ethanol (5-200 $\mu \mathrm{g} / \mathrm{mL})$. Afterward, the absorbance of different concentration of Quercetin solution was measured at $415 \mathrm{~nm}$ to obtain the calibration curve for TFC analysis.

\subsection{Statistical Analysis}

All experiments were conducted in triplicates and expressed in mean \pm standard deviation. Student's t-test was performed for this statistical analysis, and data is considered significantly different when $\mathrm{p} \leq 0.05$ for $95 \%$ confidence level.

\section{RESULTS AND DISCUSSION}

\subsection{Calibration Curve and Linearity for Quantification}
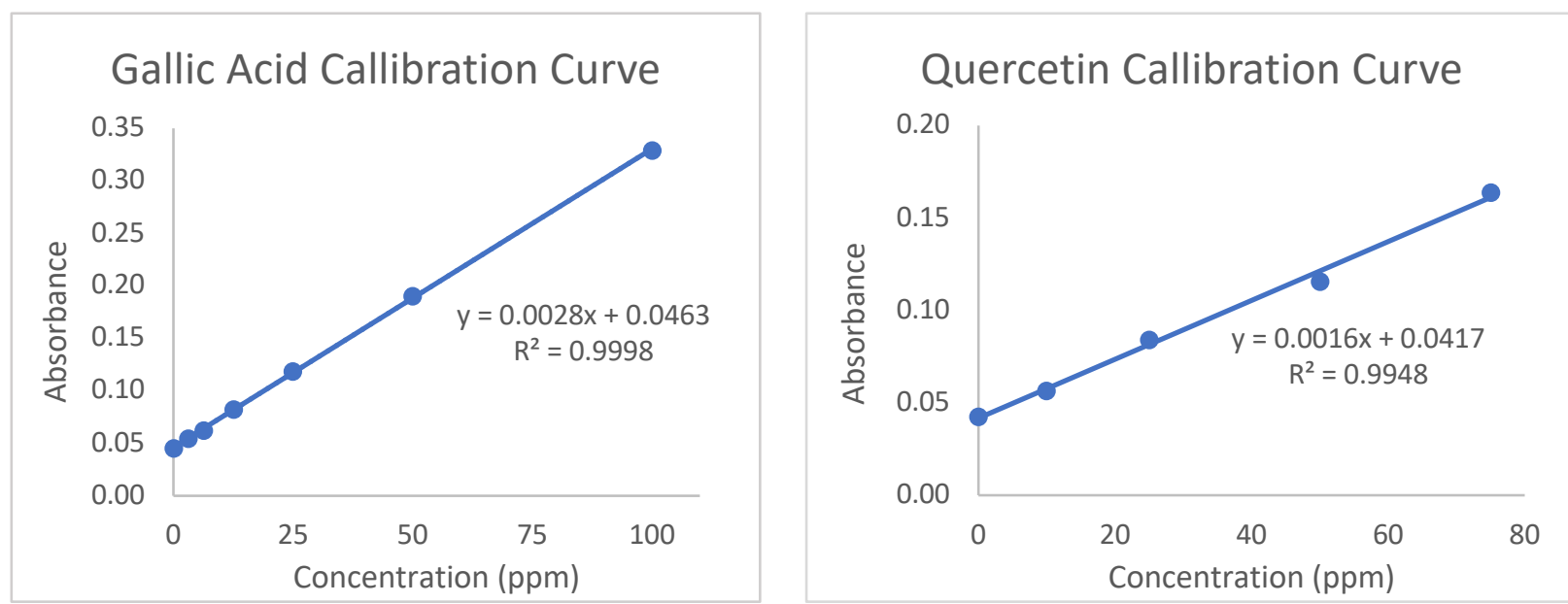

Figure 5. Gallic Acid and Quercetin Standard Calibration Curve

The calibration curve was found to be linear in the range of 0 to $100 \mathrm{ppm}$ for gallic acid and quercetin standards, as shown in Figure 5. The regression equation for gallic acid $(y=0.0028 x+0.0463)$ and quercetin $(y=0.0016 x+0.0417)$ possessed good correlation coefficients of 0.9998 and 0.9948 respectively. This shows a good linearity response for 
the method developed as the correlation coefficient of 0.99 indicates that the calculated line could explain more than $99 \%$ of the experimental data. ${ }^{18}$

\subsection{Total Polyphenolic Content (TPC)}

The bio-fermentation of Okara and BSG using Aspergillus Oryzae strains were expected to show positive nutritional enhancements. TPC and TFC assays were performed to analyze the changes in polyphenols after fermentation.

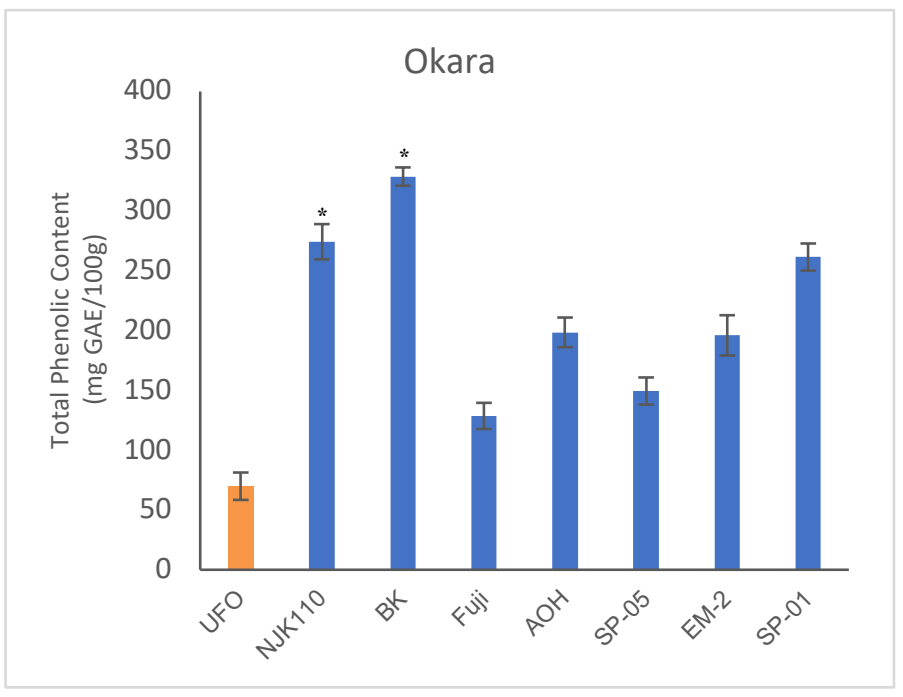

Figure 6. TPC of unfermented and fermented Okara. * indicates significant differences, $\mathrm{p} \leq 0.05$ for $95 \%$ confidence level

As shown in Figure 6, the level of phenolic contents increased post-fungal bio-fermenting Okara by Aspergillus Oryzae. Fermented Okara extracts increased by 2 to 5 -fold compared to unfermented Okara, which has $69.88 \pm 11.45 \mathrm{mg}$ GAE/100 g Okara dry weight. NJK110 and Black Koji Okara showed the most efficient fermentation, where they increased the TPC content by 4 and 5-fold, respectively. It might be due to the high citric acid produced by NJK110 and Black Koji. This suggest the dissociation of polyphenolic forms due to the citric acid hydrolyzation, which are responsible for the increase in TPC. Thus, it is possible that synergistic reaction occurred with the application of the acid, activating the transduction pathways that conduct the secondary signals produced by plants. ${ }^{19}$ Meanwhile, the fermented Okara by Fuji microbe had the lowest increase in TPC content (128.68 \pm $10.93 \mathrm{mg} \mathrm{GAE} / 100 \mathrm{~g}$ Okara dry weight) than the unfermented Okara extract. 


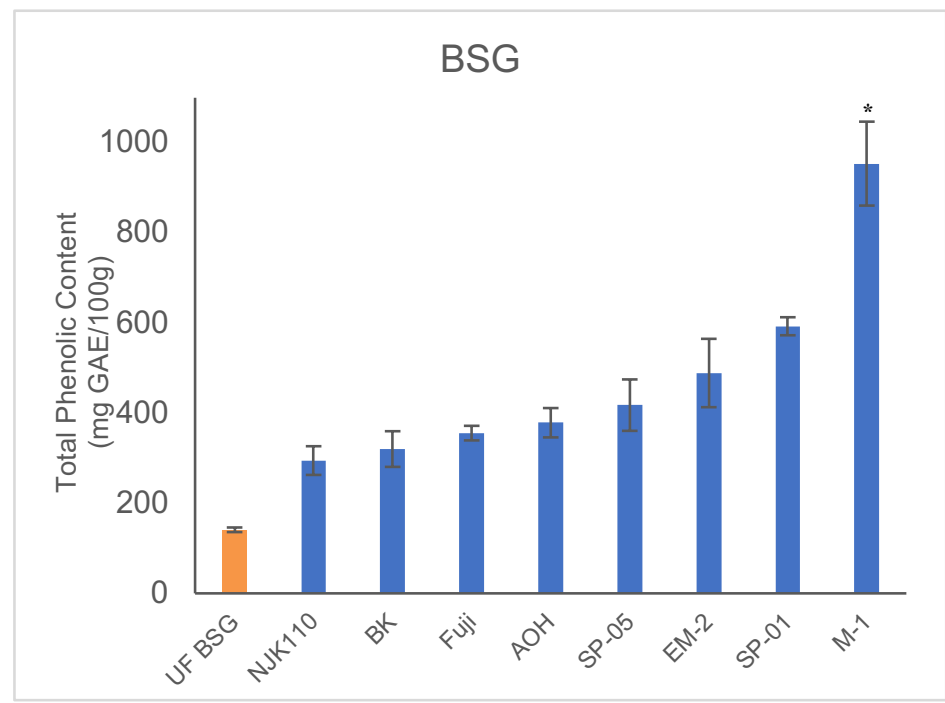

Figure 7. TPC of raw and fermented BSG. * indicates significant differences, $\mathrm{p} \leq 0.05$ for $95 \%$ confidence level

The fermented BSG results in Figure 7 also agrees with the hypothesis whereby TPC content is enhanced upon fermentation by Aspergillus Oryzae. Comparing the performance of different microbes, M-1 BSG shows the best performance in the quantification of TPC. A significant increase was observed, giving approximately a 7-fold increase compared to unfermented BSG $(141.11 \pm 5.13 \mathrm{mg}$ QE/100 g Okara dry weight). With the nature of BSG and the use of M-1 microbe, which is a potent saccharifying enzyme producer, this enzyme will gradually be inhibited at the later stage of fermentation, hence, produced higher citric acid, leading to the highest enhancement in phenolic content. ${ }^{20}$ NJK110 and Black Koji BSG had no significant change in TPC than unfermented BSG. There might be no significant contribution towards citric acid accumulation, due to the optimal amount of nitrogen available in fermentation medium, causing reduction in citric acid production. ${ }^{21}$ Fermentation period is believed to play a significant role in the changes of nitrogen available in the substrate. ${ }^{21}$

All in all, with fermentation, the proteolytic enzymes from the starter microbes will hydrolyze the complex phenolics. ${ }^{22}$ Hence, the increase in TPC values might be due to the hydrolysis of complex phenolics into smaller molecules, such as soluble-free phenols and other biologically active ones, which are readily absorbed during the fermentation process. 


\subsection{Total Flavonoids Content (TFC)}

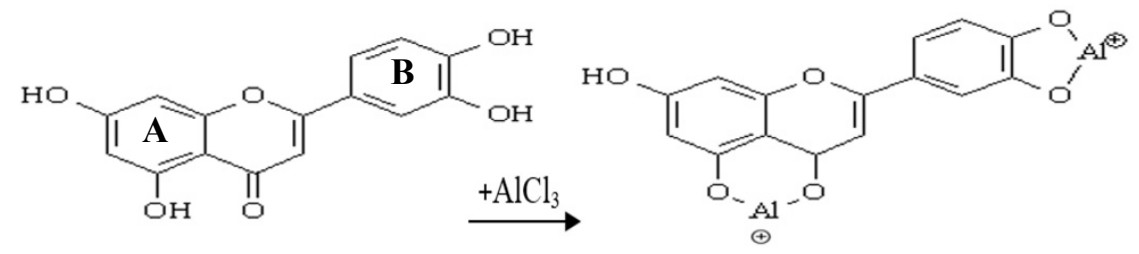

Scheme 4. Formation of Flavonoids Complex with $\mathrm{AlCl}_{3}$

Among the polyphenols are flavonoids, which have been the subject of interest due to their potential application in healthcare as natural antioxidants. The science behind TFC determination was the formation of stable acid complexes between $\mathrm{AlCl}_{3}$ and the $\mathrm{C}-4$ keto groups together with either the C-3 or C-5 hydroxyl group of flavones and flavonols. Moreover, $\mathrm{AlCl}_{3}$ helps to form acid-labile complexes with the orthodihydroxyl groups in the A and B-ring of flavonoids. ${ }^{23}$

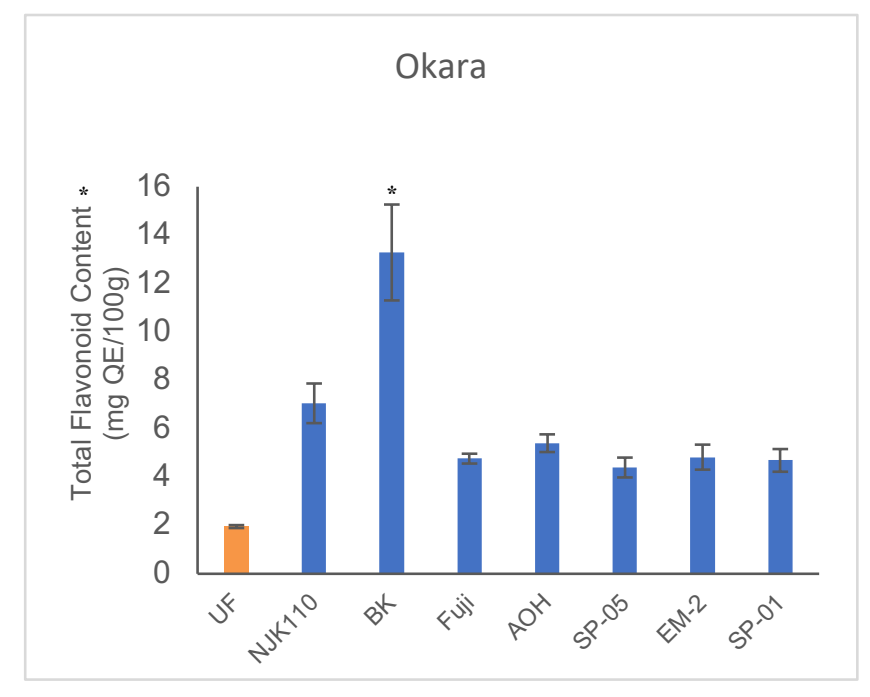

Figure 8. TFC of raw and fermented Okara. * indicates significant differences, $\mathrm{p} \leq 0.05$ for $95 \%$ confidence level

Figure 8 shows that TFC also increased after the fermentation of Okara by Aspergillus Oryzae strains. Broadly, the extent of increase in TFC for the respective fermented okara samples compared to that of unfermented okara is different from that of TPC. However, the samples fermented with NJK110 and Black Koji had the largest increase in both TPC and TFC. Fermented Okara extracts had TFC increment ranging from 2 to 13-fold compared to raw Okara. NJK110 and Black Koji Okara have the highest increase in TFC content by 7 and 13fold, respectively. It can be inferred that fermented Okara extracts had significantly better TFC compared to their unfermented equivalent. As mentioned, both NJK110 and Black Koji possess 
high citric acid, which helped to increase the synthesis of secondary metabolites, such as flavonoids, thus, higher TFC. ${ }^{19}$

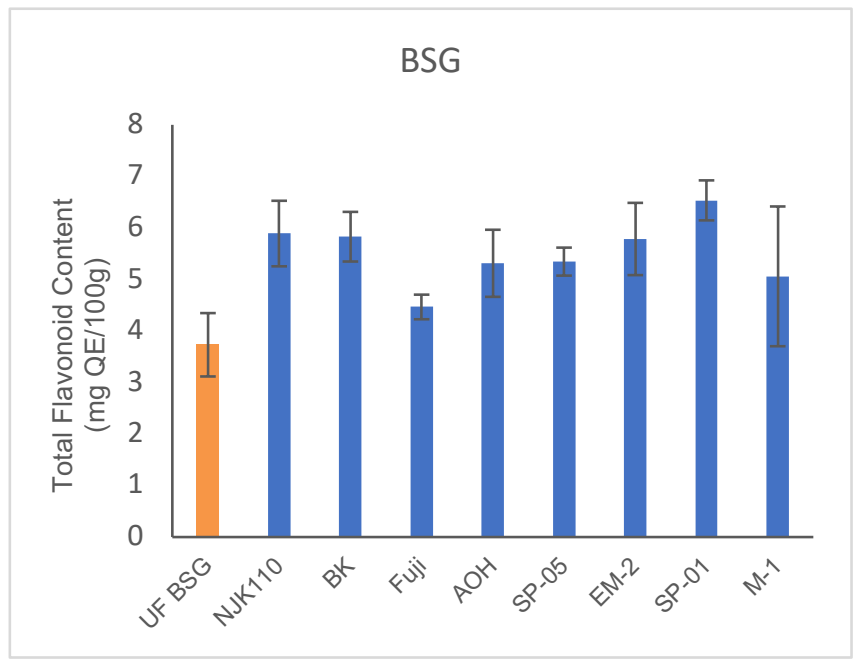

Figure 9. TFC of raw and fermented BSG.

Conversely, the TFC of fermented BSG by various microbes showed a similar increase in the flavonoid content shown in Figure 9. Raw BSG possessed $3.73 \pm 0.61 \mathrm{mg} \mathrm{QE} / 100 \mathrm{~g}$ Okara dry weight, and there was only a slight increase observed in the remaining fermented BSG, approximately 2-fold. It can be inferred that the microbes used are not as efficient in increasing Total Flavonoid Content (TFC) of BSG as compared to Okara, or rather, samples being too old, thus affect their shelf-life and reduce the nutritional content.

The increase observed in flavonoids content of Okara and BSG due to fermentation might be because of the increase in acidic value during fermentation, which liberates bound flavonoids components, hence, more bioavailable. ${ }^{24}$ The extent of increase in TFC depends on the microbes' ability in producing $\beta$-glucosidases, which were used to catalyze the release of flavonoids from substrates. Interestingly, similar trends were observed in the TPC and the TFC value of Okara, but not BSG. It might be because a majority of the flavonoids of BSG were conjugated with different types of sugars, hence, solvent solubility plays a role in dissolving flavonoids compound. ${ }^{23}$

Furthermore, solid-state fermentation has been reported to enhance the quality of amino acids. ${ }^{24}$ For that matter, an attempt to analyze amino acids analysis was made. However, due to several technical difficulties compounded by the limited time frame, the 
quantification of the amino acid content of unfermented and fermented Okara and BSG was unsuccessful.

\section{CONCLUSION AND FUTURE WORK}

It can be concluded that solid-fermentation using Aspergillus Oryzae improved the polyphenol profile of Okara and BSG. Black Koji Okara and M-1 BSG extracts showed the highest increase of 5- and 7-fold in TPC as well as 13- and 2-fold in TFC with their respective microbes. Different microbes show different effectiveness in fermenting Okara and BSG. Some microbes might be helpful in synthesizing flavonoids, but some others might be better in synthesizing other types of polyphenols. As polyphenols benefit the human body due to their natural antioxidant capacity and other bioactivities, this work should be further studied to prove the nutritional enrichment of both Okara and BSG through fermentation. The study of individual isoflavones might be useful to understand the metabolic processes activated by the bio-fermentation procedure. Moreover, to support the nutritional enhancement in fermented biomass, other nutrients (e.g., Amino Acids, Saponins, etc) could be further investigated as they are also beneficial for human health. ${ }^{25-}$ 27 


\section{REFERENCES}

(1) El Bilali, H.; Ben Hassen, T. Food Waste in the Countries of the Gulf Cooperation Council: A Systematic Review. Foods 2020, 9 (4), 463.

(2) Food waste management. Retrieved from: https://www.nea.gov.sg/our-services/wastemanagement/3r-programmes-and-resources/food-waste-management (accessed March $16,2021)$

(3) Singapore's Food Security. Retrieved from: https://agrifood.net/images/cfs43/CFS43\%20Sideevent $\% 20$ background $\% 20$ document $\% 20-\% 20$ Lessons $\% 20$ from $\% 20$ Singapore.pdf (accessed Mar 16, 2021)

(4) Food Waste. Retrieved from: https://www.towardszerowaste.gov.sg/foodwaste/ (accessed Mar 16, 2021)

(5) Mok, W. K.; Tan, Y. X.; Chen, W. N. Technology Innovations for Food Security in Singapore: A Case Study of Future Food Systems for an Increasingly Natural ResourceScarce World. Trends Food Sci. Technol. 2020, 102, 155-168.

(6) Gupta, S.; Chen, W. N. A Metabolomics Approach to Evaluate Post-fermentation Enhancement of Daidzein and Genistein in a Green Okara Extract. J. Sci. Food Agric. 2021, jsfa. 11158.

(7) Tan, Y. X.; Mok, W. K.; Lee, J.; Kim, J.; Chen, W. N. Solid State Fermentation of Brewers' Spent Grains for Improved Nutritional Profile Using Bacillus Subtilis WX-17. Fermentation 2019, 5 (3), 52.

(8) In Seong Choi, Young Gyu Kim, Ja Kyun Jung, Hyeun-Jong Bae. Bioethanol Production from Renewable Raw Materials and Its Separation and Purification: A Review. Food Technol. Biotechnol. 2018, 56 (3).

(9) Lynch, K. M.; Steffen, E. J.; Arendt, E. K. Brewers' Spent Grain: A Review with an Emphasis on Food and Health: Brewers' Spent Grain: A Review with an Emphasis on Food and Health. J. Inst. Brew. 2016, 122 (4), 553-568.

(10) Cooray, S. T.; Chen, W. N. Valorization of Brewer's Spent Grain Using Fungi SolidState Fermentation to Enhance Nutritional Value. J. Funct. Foods 2018, 42, 85-94.

(11) Li, W.; Wang, T. Effect of Solid-State Fermentation with Bacillus Subtilis Lwo on the Proteolysis and the Antioxidative Properties of Chickpeas. Int. J. Food Microbiol. 2021, $338,108988$.

(12) Queiroz Santos, V. A.; Nascimento, C. G.; Schmidt, C. A. P.; Mantovani, D.; Dekker, R. F. H.; da Cunha, M. A. A. Solid-State Fermentation of Soybean Okara: Isoflavones 
Biotransformation, Antioxidant Activity and Enhancement of Nutritional Quality. LWT 2018, 92, 509-515.

(13) WHO Cardiovascular Disease. Retrieved from: https://www.who.int/healthtopics/cardiovascular-diseases\#tab=tab_1 (accessed March 30, 2021)

(14) Chemat, F.; Vian, M. A.; Cravotto, G. Green Extraction of Natural Products: Concept and Principles. Int. J. Mol. Sci. 2012, 13 (7), 8615-8627.

(15) Ibrahim, U. K.; Salleh, R. M.; Maqsood-ul-Haque, S. N. S. Bread towards Functional Food: An Overview. ETP Int. J. Food Eng. 2015.

(16) Ibidapo, O.; Henshaw, F.; Shittu, T.; Afolabi, W. Bioactive Components of Malted Millet (Pennisetum Glaucum), Soy Residue "Okara" and Wheat Flour and Their Antioxidant Properties. Int. J. Food Prop. 2019, 22 (1), 1886-1898.

(17) Capello, C.; Fischer, U.; Hungerbühler, K. What Is a Green Solvent? A Comprehensive Framework for the Environmental Assessment of Solvents. Green Chem. 2007, 9 (9), 927.

(18) Silva, L.; Pezzini, B.; Soares, L. Spectrophotometric Determination of the Total Flavonoid Content in Ocimum Basilicum L. (Lamiaceae) Leaves. Pharmacogn. Mag. 2015, $11(41), 96$.

(19) Preciado-Rangel, P. The Effect of Citric Acid on the Phenolic Compounds, Flavonoids and Antioxidant Capacity of Wheat Sprouts. Rev. Fac. Cienc. Agrar. 2018, 9.

(20) 石贵阳; 张梁; 蒋小东; 孙福新; 丁重阳; 顾正华; 宗伟刚; 胡志杰; 蒋建伟. Method for Fermenting and Preparing Citric Acid by Adding Saccharifying Enzyme. CN101942487A, January 12, 2011.

(21) Pathania S., Sharma S., Kumari K. Solid state fermentation of BSG for citric acid production. Ind. J. Nat.Product. Res. 2018, 9, 70-74.

(22) Adebo, O. A.; Gabriela Medina-Meza, I. Impact of Fermentation on the Phenolic Compounds and Antioxidant Activity of Whole Cereal Grains: A Mini Review. Molecules 2020, 25 (4), 927.

(23) Sepahpour, S.; Selamat, J.; Abdul Manap, M.; Khatib, A.; Abdull Razis, A. Comparative Analysis of Chemical Composition, Antioxidant Activity and Quantitative Characterization of Some Phenolic Compounds in Selected Herbs and Spices in Different Solvent Extraction Systems. Molecules 2018, 23 (2), 402. 
(24) Adetuyi, F. O.; Ibrahim, T. A. Effect of Fermentation Time on the Phenolic, Flavonoid and Vitamin C Contents and Antioxidant Activities of Okra (Abelmoschus Esculentus) Seeds. Niger. Food J. 2014, 32 (2), 128-137.

(25) Kumar, V.; Rani, A.; Husain, L. Investigations of Amino Acids Profile, Fatty Acids Composition, Isoflavones Content and Antioxidative Properties in Soy Okara. Asian J. Chem. 2016, 28 (4), 903-906.

(26) Kamble, D. B.; Rani, S. Bioactive Components, in Vitro Digestibility, Microstructure and Application of Soybean Residue (Okara): A Review. Legume Sci. 2020, 2 (1).

(27) Pérez-López, E.; Mateos-Aparicio, I.; Rupérez, P. Determination of Soluble Dietary Fibre Content of Okara Treated with High Hydrostatic Pressure and Enzymes: A Comparative Evaluation of Two Methods (AOAC and HPLC-ELSD). J. Food Sci. Technol. 2017, 54 (5), 1333-1339. 